\title{
Es gibt kein Zurück - schauen wir nach vorne
}

\section{Bettina Wölnerhanssen}

Präsidentin Forum Junger Chirurgen
Korrespondenz:

Dr. med. Bettina Wölnerhanssen Tiefengrabenstrasse 43

CH-4102 Binningen

bewoe@bluewin.ch

\section{Sehr geehrter Herr Professor Gemsenjäger}

Gerne möchten wir Ihre Bemerkungen zur Ausbildung aus Sicht der jungen Generation von Chirurgen kommentieren. In vielen Punkten sprechen Sie uns aus dem Herzen. Allerdings hat sich sowohl gesellschaftlich als auch in der Chirurgie einiges verändert:

Die Chirurgie hat sich in all ihren Untergebieten rasant entwickelt. Es ist kaum mehr möglich, das ganze Spektrum der Chirurgie mit fortschreitender Komplexität der verschiedenen Behandlungsstrategien in gleichbleibend hoher Qualität anzubieten. Hier gilt es, von einem alten Zopf - dem Allrounder Abschied zu nehmen. Ein virtuoser Laparoskopiker, der auch bei den neuesten Osteosynthesetechniken mitreden kann, dürfte Seltenheitswert erreichen. Das Management eines Rektumkarzinomes setzt anderes Know-how voraus als das einer Femurfraktur.

Das Medizinstudium bringt mehr weibliche Abgängerinnen hervor, und die vielzitierte Feminisierung hat auch die Chirurgie erreicht. Die Konsequenzen sind unter anderem diskontinuierliche Lebensläufe: ein Curriculum ist bei wiederholter Mutterschaft durch Arbeitsunterbrüche und Zeiten von reduziertem Arbeitspensum geprägt. Nachwuchsmangel in der Chirurgie ist eine Tatsache. Die Frauen werden in der Chirurgie gebraucht. Durch gezielte Förderung und Angebot von familientauglichen Pensen (z.B. Weiterbildung in einer Subspezialität, was auch in Teilzeit möglich ist), sollte die Chirurgie attraktiv gemacht und ein Abbruch der Weiterbildung aus Mangel an Perspektive verhindert werden.

Die Gesellschaft hat auch das Berufsbild der Männer massgebend verändert. War es früher vielleicht noch möglich, sich nur dem Beruf zu widmen und die Haus- sowie Familienarbeit an die Ehepartnerin zu delegieren, so wird heute erwartet, dass beide Ehepartner sich in allen Arbeitsbereichen beteiligen. Die jungen Männer weigern sich, ihre Kinder nur schlafend zu erleben, sie möchten ihren Nachwuchs ebenfalls betreuen, und der Wunsch nach Teilzeitarbeit kommt auch bei Männern auf.

Die Einführung der 50-Stunden-Woche wurde meist ohne flankierende Massnahmen durchgesetzt: Anstatt den Arbeitsinhalt zu optimieren und so Zeit einzusparen, wurden schlicht mehr Arbeitskräfte eingestellt, die sich nun die gleiche Anzahl Operationen teilen müssen. Administrative Aufgaben sowie viele nichtärztliche Tätigkeiten haben klar zugenommen. In Analogie zu Ihren Ausführungen zum Neocortex

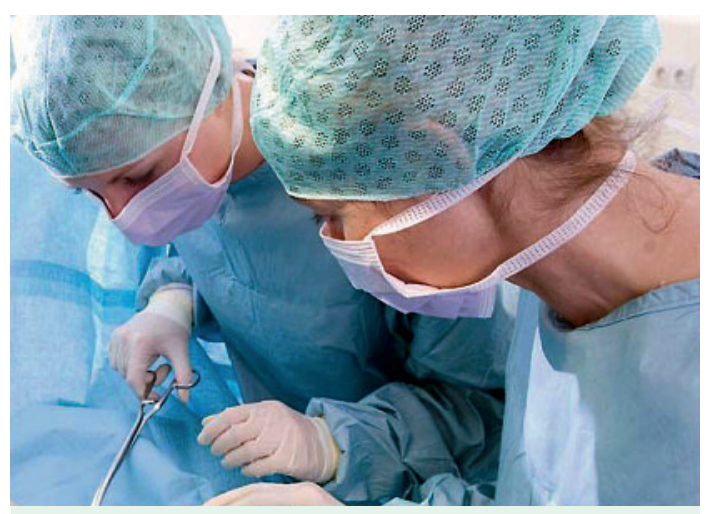

Die «Feminisierung» der Medizin hat auch die Chirurgie erreicht.

dürfte die Tastatur eine wesentliche Abbildung im entsprechenden Gebiet erfahren haben! Das Zuschauen im OP ist nicht mangels Interesse, sondern wegen Verpflichtungen fern der chirurgischen Kernkompetenz nur mehr selten möglich. Eine Rückkehr zu längeren Arbeitszeiten lehnen wir ab. Diese garantieren keinen vernünftigen Arbeitsinhalt (man kann auch 70 Stunden administrativ tätig sein) und stehen im Konflikt mit der für unsere Generation wichtigen «Work-Life-Balance». Hierbei meinen wir vor allem mehr Zeit für Familien- und Hausarbeit und nicht (wie oft unterstellt) fürs Faulenzen.

All dies ist nicht rückgängig zu machen, ob uns das passt oder nicht. Die Chirurgie wird nicht wieder einfacher. Die Frauen werden gebraucht, und um Teilzeitstellen kommen wir nicht herum. Den jungen Männern kann man keine alte Rolle aufzwingen, sie absolvieren zu Hause Überstunden. Das Arbeitszeitgesetz wird richtigerweise nicht aufgehoben. Aber all diese Veränderungen zusammen mit der von Ihnen geschilderten Wichtigkeit des technischen Trainings führt unweigerlich zum Schluss, dass die chirurgische Ausbildung nicht einfach nach altem Muster beibehalten werden kann, wenn weiterhin eine hohe Versorgungsqualität erwünscht ist. Die Lösungen heissen:

- Subspezialisierung;

- Optimierung des Arbeitsinhalts;

- Ausnutzen virtueller Medien sowie Trainingskurse an Modellen und Leichenpräparaten.

Ob die Zeiten früher besser waren (wie schon von den Römern behauptet)? Sicher waren sie anders. Es gibt aber kein Zurück. Schauen wir voller Tatendrang in die Zukunft! 